\title{
Real Burst Traffic Amplification in Optically Gain Clamped Amplifier
}

\author{
K. Ennser ${ }^{1}$, S. Taccheo ${ }^{1,2}$, D. Careglio ${ }^{3}$, J.Solé-Pareta $^{3}$ and J. Aracil ${ }^{4}$ \\ 1: Institute of Advanced Telecommunications, Swansea University, Swansea SA2 8PP, UK, e-mail: k.ennser@swansea.ac.uk \\ 2: (also with) Dipartimento di Fisica Politecnico di Milano, Italy. \\ 3: Universitat Politècnica de Catalunya, Jordi Girona, 1-3, 08034 Barcelona, Catalunya, Spain. \\ 4: Universidad Autonoma de Madrid, Ciudad Universitaria de Cantoblanco, 28049 Madrid, Spain.
}

\begin{abstract}
Optical burst amplification in a gain-stabilized amplifier is theoretically investigated using real burst traffic data. The results show that excellent performance are obtained for WDM transmission with negligible interplay due to burst arrival statistics.

(C)2008 Optical Society of America

OCIS codes: : (060.2320) Fiber optics amplifiers and oscillators; (060.4510) Optical communications.
\end{abstract}

\section{Introduction}

Optical burst transmission has been pointed out as a promising way to implement IP traffic over WDM and to guarantee payload transparency as well as efficient bandwidth utilization with respect to other solutions [1]. However the optical amplifier (OA) gain should be stabilized to avoid burst distortions due to input power variation and accumulation in cascaded amplified network. This problem will be obviously increased in a WDM multichannel system where gain of one channel will change according with the burst transmission on all other channels, and still electronic feedback generates strong gain variation after several link stages [2]. Recently we have applied the optically gain clamped (OGC) technique to stabilize the amplifier to optical burst transmission and we have noticed a complex behavior in case of burst sequences with frequency related to the natural device relaxation oscillation frequencies [3,4], with some analogies with extremely low gain laser configuration [5].

In this paper, to evaluate OGC-OA performance in real system, we report results on the dynamics of optically gain clamped amplifier in real burst data traffic. The burst data is collected from a tailor made testbed and processed to investigate the impact of optical amplifier dynamics. Several scenarios are studied and excellent performance is obtained using OGC scheme. The interplay between burst arrival time and OGC-OA dynamics is studied. For both WDM and single-channel systems. Cascaded OGC-OAs are considered to estimate the accumulated gain variation and design robustness is discussed. The results rely on a program validated extensively [4,6] and we are confident represents an reliable picture of OGC-OA performance with burst traffic.

\section{Real burst data collection}

We consider a scenario where several client networks are attached to a single OBS node which performs packets aggregation and bursts generation. For the client traffic we use real packet traces captured with a tailor-made measurement platform designed to operate at gigabit speed without packet losses and ns-precision in the packet timestamp measurements. The point of measurement is a pair of Full-Duplex Gigabit Ethernet links (two per each traffic direction) that connects the Catalan R\&D network (about 50 Universities and Research Centers) with the Spanish R\&D

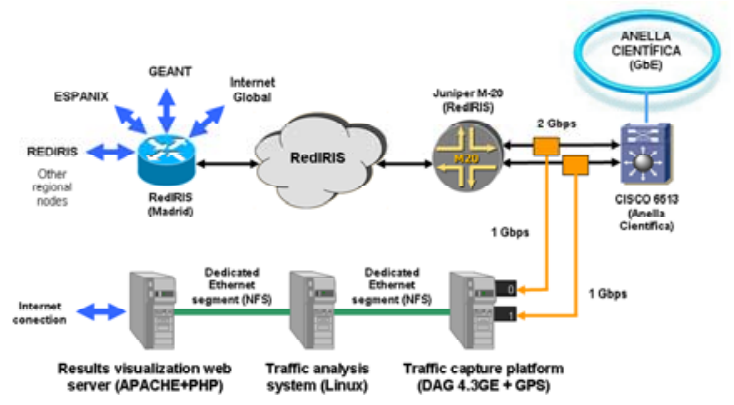

Fig. 1: Experimental testbed to collect burst data.

RedIris network and to the global Internet [7]. A hybrid timer-length threshold burst assembler [8] is used to aggregate the packets; the timer threshold is set to $5 \mathrm{~ms}$ and the maximum burst length to 250 kbytes. We assume that the OBS network domain is composed of 30 nodes; the destination of the bursts is therefore obtained aggregating the IP addresses of the packet traces according to the geographical location.

\section{Optical burst amplification}

In order to investigate the optical burst amplification we use a two-level equation set to simulate the dynamics of the optically gain clamped Erbium-doped fiber amplifier (OGC-EDFA) [9]. This model is simpler than the one used in Ref.4,6 but, after comparison, proved to be effective for this kind of simulation and was therefore chosen to reduce the computational time. In this model the OGC configuration is described as a laser system. As consequence the 


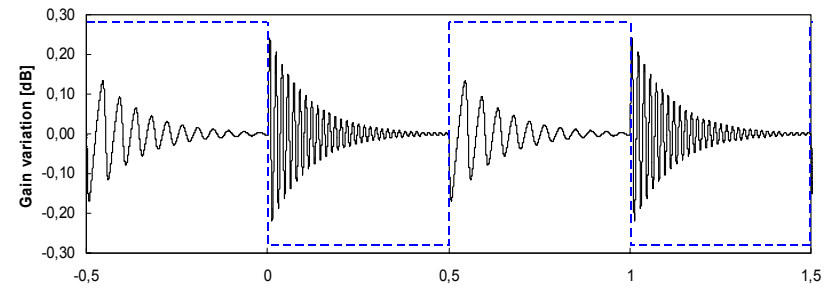

Fig. 2: Gain variation under on/off of all channels pump flux is expressed as a function of laser threshold pump flux which relates as $\mathrm{P}_{\mathrm{p}}=\mathrm{x} \mathrm{P}_{\mathrm{P}, \mathrm{th}}$. The amplifier is assumed to have $20 \mathrm{~dB}$ gain and the cavity length is $21 \mathrm{~m}$. The maximum total input power is $-1 \mathrm{dBm}$ when all WDM burst channels are simultaneously entering the amplifier. The pump power level is set to $\mathrm{x}=1.15$, i.e. at $15 \%$ higher level than for unclamped EDFAs [9]. Fig. 2 shows the OGC-OA dynamics when all channel are switched on/off.

The gain excursion is limited to below $0.25 \mathrm{~dB}$ and this is the maximum gain variation related to eventual sudden failure or network restoration. The typical laser relaxation oscillation frequency (ROF) for full channel load (on) or no channel (off) is $22 \mathrm{kHz}$ and $66 \mathrm{kHz}$, respectively. This set the limits of ROFs characteristics of our

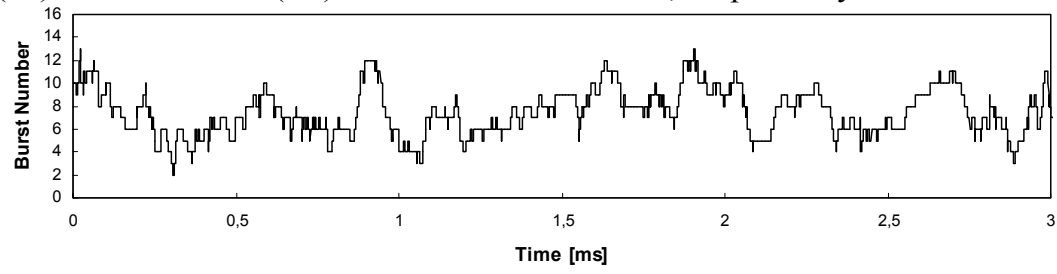

Fig. 3: Burst number along time (-1 dBm total power when all 16 channels)
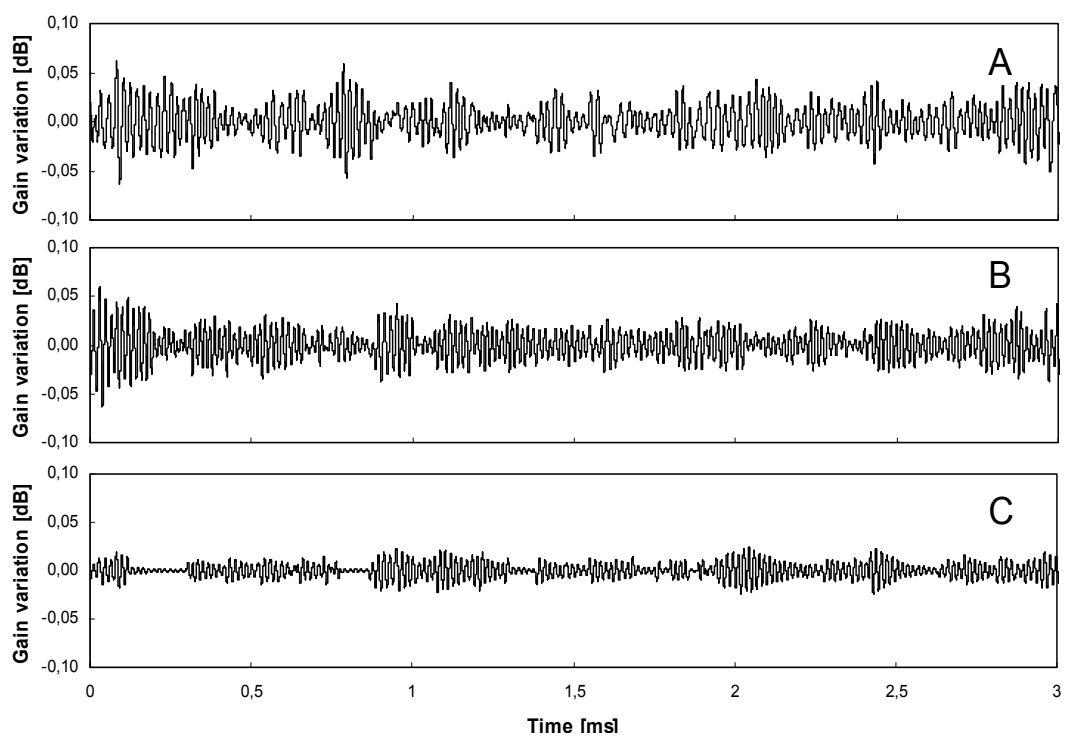

Fig. 4: Gain variation in case of (A) 16 channels and $x=1.15$; (B) 16 channel and $\mathrm{x}=1.5$; (C) 4 channel $\mathrm{x}=1.15$ amplifier. In a previous work we have shown that if the burst frequency interplay with the characteristics OGC-OA relaxation oscillation frequencies we may have enhanced oscillation, with interplay starting from $v_{\text {on }} / 3$ to about 2 times $v_{\text {off }}$, corresponding in our case to 7 $\mathrm{kHz}$ to $100 \mathrm{kHz}$ interval [6]. We have also demonstrated that high gain variation were induced after a few burst sequence [4]. The real data burst distribution fall well within this interval, however different channel will combine in a random mode to generate a total power time variation as stated below.

The aim of this work is to evaluate the effect on OGC-OA of real burst data traffic and verify if there are still instabilities or oscillations due to the interplay between burst and OGC-OA dynamics [6]. To address this issue we collect a $95 \mathrm{k}$ burst trace (duration $>14$ sec) in the testbed described in the prior section. We elaborate a

WDM system with 16 channel by using uncorrelated data in different time slot for different channel to generate an about $1 \mathrm{~s}$ long trace. To enable an easy visualization and significant representation of the input data here we show 3-ms trace. Fig. 3 shows the total power (i.e. sum of all burst channel). We see that we never have less than 2 channels and more than 13 channels at the same time. Along the $1 \mathrm{~s}$ trace we had a minimum and maximum of 1 and 15 channel respectively. We also note that the average power is about $-4 \mathrm{dBm}$ ( 8 channel out of 16) and we miss large variation of channel number in times comparable with laser dynamics $(<50 \mu \mathrm{s})$. This suggest that the OGC-OA will work well above threshold and is not subject to abrupt input power variation like in the on/off case.

Fig. 4a shows the gain variation induce by the trace of Fig. 3 for a OGC-OA working at $x=1.15$. Figure $4 \mathrm{~b}$ shows the same results but in case of $x=1.5$. We do not see any appreciable variation as the OGC-OA in burst mode works with average power of $-4 \mathrm{~dB}$ and therefore is typically far more above threshold than $\mathrm{x}=1.15$. However we note that at same time interval in Fig. $4 \mathrm{~b}$ sometime we have stronger gain variations than in Fig.4a despite a larger $\mathrm{x}$ value. This is simply due to the fact that at different $x$ value corresponds different ROF and therefore the burst sequence 


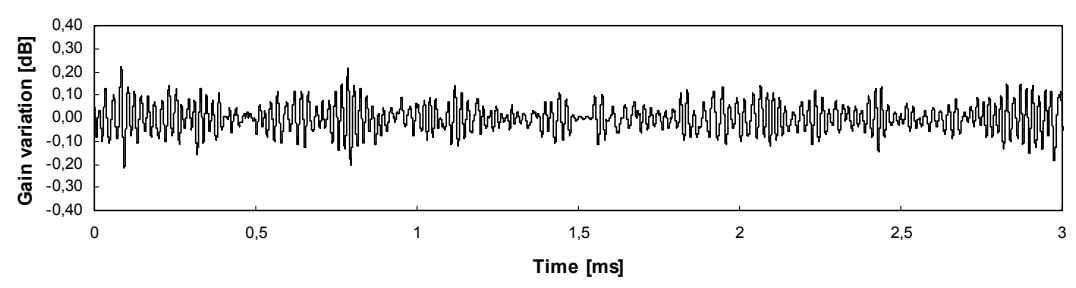

Fig. 5: Gain variation after four cascaded OGC-EDFAs for 16 channels and $\mathrm{x}=1.15$.
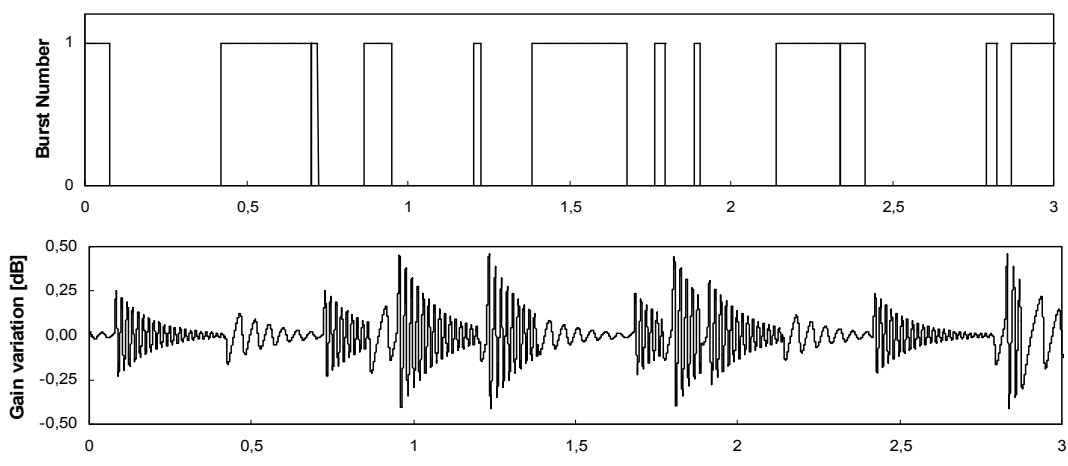

Fig. 6: (a) Burst trace (b) Gain variation for input power of $-1 \mathrm{dBm}$ and $\mathrm{x}=1.15$.

OGC-OA will effectively perform in case of WDM burst traffic.

A different scenario is if the OGC-OA is used in few channel link with high channel power.. As example we simulate a link with one channel link with $-1 \mathrm{dBm}$ input. Figure $6 \mathrm{a}$ illustrates the trace and Fig. $6 \mathrm{~b}$ shows the dynamics of gain variation. As results we note the interplay between burst sequence interarrival times and OGC-OA ROFs. In fact we have stronger gain variation than in the on/off case (see Fig.2) as expected according to experimental verification in $[4,6]$. The real traffic data may therefore induce significant gain variation. To minimize the gain excursions, the OGC-EDFA should operate with a larger $\mathrm{x}$ value of about 1.5 or with shifted ROFs [6].

\section{Conclusions}

We have demonstrated that OGC-OA are suitable for optical burst transmission. Excellent performance have been calculated using real data traffic and an accurate model able to well describe experimental amplifier dynamic results. We have still noticed an effect of interplay between burst and OGC-OA dynamics but no strong gain fluctuations due to the low average power in burst traffic. We have simulated a cascade of OGC-OA and we found an almost linear accumulation of gain dynamics effect with a maximum gain excursion of $0.5 \mathrm{~dB}$ after 4 stages. We therefore prove that OGC-OA is suitable for burst traffic.

Acknowledgments: This work was partially supported by the European Union through the Welsh Assembly Government, the European Union funded Network of Excellence E-photon1+ and COST291.

\section{References}

[1] Yu-Li Hsueh, J. Kim, Ching-Fong Su, R. Rabbat, T. Hamada, C. Tian and L.G. Kazovsky, "Traffic grooming on WDM rings using optical burst transport," J. Lightwave Technol., vol. 24, pp. 44-53, 2006.

[2] A. Lieu, C. Tian, T. Naito, "Transmission and interactions of WDM burst signals in cascaded EDFAs," OFC'06, USA, paper OtuD5.

[3] S. Taccheo, A. Festa G. Della Valle and K. Ennser "Gain-stabilized Erbium-Doped Waveguide Amplifier for Burst Transmission", IEEE Photon. Technol. Lett., 19, 97-99 (2007)

[4] S. Taccheo, G. Della Valle, A. Festa and K. Ennser, "Amplification of Optical Bursts in Gain-Stabilized Erbium-Doped Optical Amplifier", OFC2007, USA, paper OMN3

[5] L. Luo, T. J. Tee and P. L. Chu, "Chaotic behavior in erbium-doped fiber-ring lasers ,” J. Opt. Soc. Am. B, vol. 15, pp. 972-978, 1998.

[6] G. Della Valle, A. Festa, S. Taccheo, K. Ennser and J. Aracil " Nonlinear dynamics induced by burst amplification in optically gainstabilized erbium-doped amplifiers", Optics Letters, 32, 903-905, 2007

[7] P. Barlet, J. Sole Pareta, J. Barrantes, E. Codina, J. Domingo, "SMARTxAC: A Passive Monitoring and Analysis System for High-Speed Networks", in Proc. Terena Networking Conference. May 2006, Catani, Italy.

[8] X. Yu, J. Li, Y. Chen, and C. Qiao, "Traffic statistics and performance evaluation in Optical Burst Switching networks", IEEE/OSA Journal of Lightwave Technology, vol. 22, no. 12, 2004.

[9] K. Ennser, G. Della Valle, M. Ibsen, J. Schmulovich and S. Taccheo, "Erbium-doped waveguide amplifier for reconfigurable WDM metro networks", IEEE Phot. Tech. Lett., vol. 17, pp. 1468-1460, 2005. 